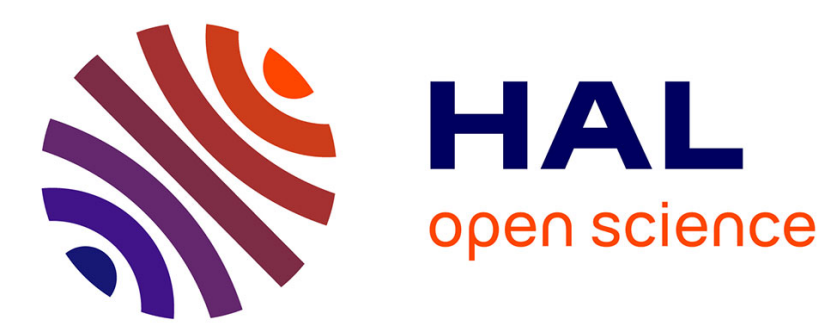

\title{
Focalisation en émission-réception en milieu inhomogène: le concept de filtre adapté
}

\author{
C. Dorme, M. Fink
}

\section{To cite this version:}

C. Dorme, M. Fink. Focalisation en émission-réception en milieu inhomogène: le concept de filtre adapté. Journal de Physique IV Proceedings, 1994, 04 (C5), pp.C5-897-C5-900. 10.1051/jp4:19945196 . jpa-00252879

\section{HAL Id: jpa-00252879 https://hal.science/jpa-00252879}

Submitted on 1 Jan 1994

HAL is a multi-disciplinary open access archive for the deposit and dissemination of scientific research documents, whether they are published or not. The documents may come from teaching and research institutions in France or abroad, or from public or private research centers.
L'archive ouverte pluridisciplinaire HAL, est destinée au dépôt et à la diffusion de documents scientifiques de niveau recherche, publiés ou non, émanant des établissements d'enseignement et de recherche français ou étrangers, des laboratoires publics ou privés. 


\title{
Focalisation en émission-réception en milieu inhomogène : le concept de filtre adapté
}

\author{
C. DORME et M. FINK
}

Laboratoire Ondes et Acoustique, Université Paris VI, E.S.P.C.I., 10 rue Vauquelin, 75231 Paris cedex 05, France

\begin{abstract}
Time reversal of ultrasonic fields is a mean to focus through inhomogeneous media on a reflective target. In previous papers, we have shown that in contrast to time delay focusing techniques, that can only correct distortions produced by a thin aberrator located close to the transducer array, TRM focusing is able to compensate for distortion independently of the aberrator position. We have also shown that the TRM focusing is optimal as it realizes the spatial-temporal matched filter to the propagation transfer function through inhomogeneous media. It is a self-adaptive technique that compensates for any geometrical distortions of the array structure as well as for distortions due to the propagation through inhomogeneous media. However, TRM focusing has only been described in the transmit mode. The extension of this matched filter focusing technique in the receive mode is presented in this paper. This new focusing technique can be achieved using adaptive convolution techniques instead of delay line techniques. Theoretical and experimental results will be presented.
\end{abstract}

\section{Focalisation en émission.}

L'instrumentation actuelle de l'imagerie échographique convient généralement d'une vitesse du son constante dans le milieu à explorer, typiquement $1540 \mathrm{~m} / \mathrm{s}$. En réalité, les variations internes de densité et de compressibilité du milieu inhomogène provoquent de sévères distorsions d'un front d'onde se propageant dans le milieu. Dès lors, la focalisation directe en un point précis par émission d'un front d'onde cylindrique dans le cas d'un réseau de transducteurs 2D (sphérique dans le cas d'un réseau 3D) s'avère inefficace.

Des méthodes adaptatives de correction des aberrations de phase estiment, par intercorrélation entre voies voisines du réseau, le retard à appliquer à chaque voie émettrice. Ces méthodes parviennent à refocaliser un champ de pression sur un réflecteur présent dans le milieu dans le cas d'aberrations de phase uniquement. Nous avons déjà montré $[1,4,5]$ que dans le cas d'un écran de phase éloigné du réseau de transducteurs ou d'inhomogénéités réparties dans le milieu, la diffraction entrainait non seulement des aberrations de phase, mais aussi des variations d'amplitude et de forme temporelle du signal. La signature d'une source ponctuelle dans un tel milieu ne consiste donc pas en un simple front d'onde distordu, mais en un véritable volume d'onde associé. Pour pallier ces effets de propagation, le retournement temporel propose de réémettre dans une chronologie inversée tout le volume d'onde reçu provenant du réflecteur. Pour cela, le retournement temporel procède en trois étapes. Le milieu est tout d'abord insonifié par un large front d'onde divergent; le réflecteur génère alors un champ de pression rétrodiffusé se propageant jusqu'au réseau de transducteurs qui l'enregistre; ce champ est ensuite retourné temporellement, puis réémis dans le milieu inhomogène pour focaliser optimalement sur le réflecteur. La Figure 1 illustre cette procédure de focalisation en émission.

Dans le cas d'un milieu satisfaisant la première approximation de Born (diffusion simple), un miroir à retournement temporel (M.R.T.) est capable de corriger les effets de diffraction engendrés par un écran aberrateur, et ceci où que soit placé l'écran dans le faisceau ultrasonore. L'avantage du retournement temporel par rapport aux autres méthodes de focalisation en émission en milieu inhomogène va, dans 
un premier temps, être montré expérimentalement, puis dćmontré par le concept de filtre adapté.

\subsection{Résultats expérimentaux.}

Les expériences effectuées l'ont été à l'aide d'une barrette linéaire de transducteurs de fréquence centrale $3 \mathrm{MHz}$. L'aberrateur est constitué par une couche de silicone placée, pour les différentes expériences, contre la barrette puis à $25 \mathrm{~mm}$ et à $50 \mathrm{~mm}$. Les diagrammes de directivité présentés dans toute la suite de l'article sont réalisés à $90 \mathrm{~mm}$ de la barrette (plan focal) par un hydrophone "aiguille" dont l'extrémité de faible diamètre sert à la fois d'instrument de mesure et de cible réflectrice.

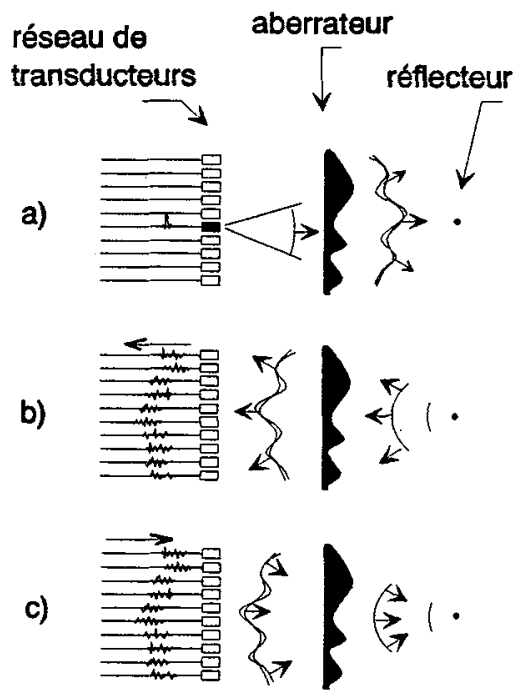

Fig. 1. La focalisation par retournement temporel requiert 3 étapes : l'émission d'un front d'onde (a), l'enregistrement du champ rétrodiffusé (b), la rémission du champ retoumé temporellement pour focaliser optimalement sur la cible réflectrice (c).

L'hypothèse d'une vitesse du son connue et uniforme dans le milieu conduit à l'émission de front d'ondes cylindrique pour focaliser au point désiré. La présence d'une couche aberratrice d'épaisseur variable et de vitesse du son différente entre le réseau et le point focal influence très défavorablement la focalisation. L'effet principal est un élargissement du faisceau ultrasonore ainsi qu'une forte remonté des lobes secondaires (voir Figure 2(a)). Des méthodes de retards adaptatifs peuvent espérer retrouver un diagramme de directivité se rapprochant de celui mesuré sans aberrateur $[2,3]$. La figure 2(b) nous montre que ce procédé n'est capable de corriger que les effets de propagation équivalents à des retards, c'est à dire ceux dus à l'épaisseur variable de l'aberrateur quand il se trouve accolé à la barrette.


Fig. 2. Diagrammes de directivité obtenus dans le plan focal par émission d'une loi de phase cylindrique (a), d'une loi de retards adaptatifs (b) et du retoumé temporel (c). L'aberrateur est successivement placé contre le réseau, à $25 \mathrm{~mm}$ puis à $50 \mathrm{~mm}$.

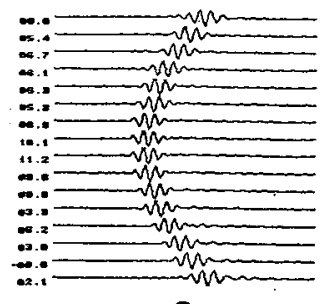

a

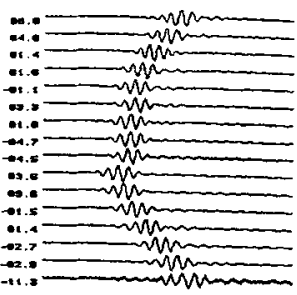

b

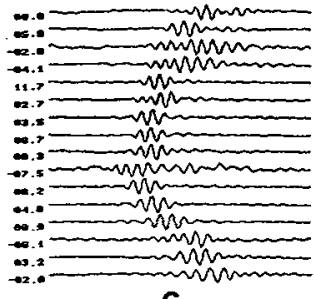

c

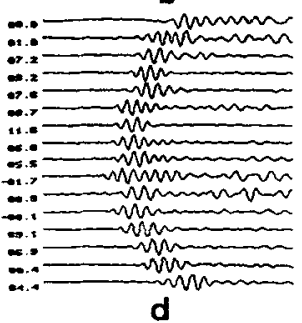

Fig. 3. Echos du réflecteur enregistrés sans aberrateur (a), puis avec l'aberrateur placé contre le réseau (b), à $25 \mathrm{~mm}$ (c) et à 50 $\mathrm{mm}$ (d) du réseau de transducteurs. 
Dans ce cas simple, les signaux reçus sur les transducteurs sont identiques à un retard près. L'écho reçu, fonction de Green du réflecteur, peut alors être assimilé à une surface d'onde (voir Figure 3(a)-(b)), ce qui n'est plus possible lorsque l'aberrateur est éloigné des transducteurs (voir Figure 3(c)-(d)). C'est pourquoi, pour focaliser optimalement sur le réflecteur, il est nécessaire de réémettre la signature du réflecteur dans son intégralité. Ainsi, le retournement temporel s'affranchit des effets de diffraction en s'adaptant automatiquement au point focal désiré. L'amélioration apportée par cette technique est évidente au vu de la Figure 2(c). Le système corrige les effets de propagation où que soit l'aberrateur en réalisant automatiquement un filtre adapté à la fonction de transfert de propagation du milieu inhomogène.

\subsection{Filtre adapté en émission.}

Chaque transducteur $\mathrm{E}_{\mathrm{i}}$ reçoit du réflecteur, considéré comme ponctuel et idéal, le signal :

$$
r_{i}\left(r_{0}, t\right)=h_{i}\left(r_{0}, t\right) \otimes h_{i}^{\text {ae }}(t)
$$

où $h_{i}\left(r_{0}, t\right)$ est la réponse impulsionnelle de diffraction du milieu pour une impulsion se propageant de $r_{0}$ au transducteur $E_{i}$ et $h_{i}^{\text {ac }}(t)$ la réponse acousto-électrique de ce transducteur (considérée équivalente à la réponse électro-acoustique). Le signal électrique retransmis au transducteur est le retourné temporel du signal reçu, soit:

$$
t_{i}\left(r_{0}, t\right)=r_{i}\left(r_{0}, T-t\right) .
$$

Le signal acoustique observé au point $r_{0}$ a alors pour expression:

$$
\begin{gathered}
a_{i}\left(r_{0}, t\right)=t_{i}\left(r_{0}, t\right) \otimes h_{i}^{a e}(t) \otimes h_{i}\left(r_{o}, t\right) . \\
a_{i}\left(r_{0}, t\right)=r_{i}\left(r_{0}, T-t\right) \otimes r_{i}\left(r_{0}, t\right) .
\end{gathered}
$$

Ce signal est symétrique avec un maximum au temps T quel que soit le transducteur. Ceci est dû à la théorie du filtre adapté qui, pour un système linéaire de réponse impulsionnelle $h(t)$, donne l'entrée $i(t)=h(-t)$ maximisant le signal de sortie pour une énergie d'entrée donnée.

Ainsi, le champ de pression résultant au point $r_{0}$ est l'interférence constructive de tous les signaux acoustiques individuels ayant leur maximum au temps $T$ en $r_{0}$. L'ensemble de signaux $t_{i}\left(r_{0}, t\right)$ constitue donc une banque de données parfaitement adaptée au point réflecteur. En tout autre point $r_{1}$, le signal acoustique mesuré décroît fortement puisque la base de données n'est plus adaptée aux réponses impulsionnelles de diffraction $h_{i}\left(r_{1}, t\right)$, entraînant des interférences destructives.

Le concept de filtre adapté permet de comprendre comment un M.R.T. focalise optimalement en émission. Nous allons montrer comment la base de données acquise permet de réaliser également une focalisation optimale en émission-réception.

\section{Focalisation en émission-réception.}

Nous avons vu que le retournement temporel s'affranchit physiquement des effets de propagation en milieu inhomogène. L'approche par filtre adapté permet d'illustrer deux principes essentiels à cette méthode: le principe de réciprocité, valable aussi en milieu inhomogène, et linvariance de l'équation de propagation après inversion de la variable temporelle. Ce concept de filtre adapté a aussi été développé pour focaliser en réception.

\section{focalisation en émission}



focalisation en réception

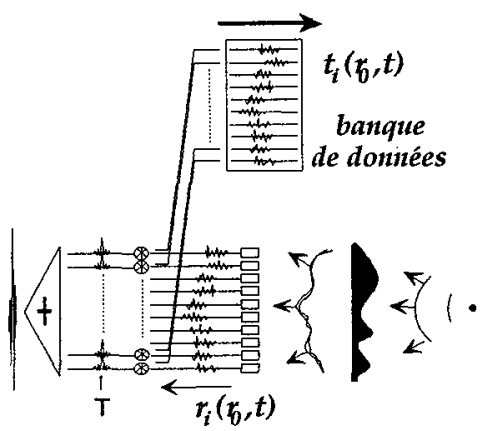

Fig. 4. Procédures de focalisation par retournement temporel. La signature du réflecteur est enregistrée dans une banque de donmées qui sert à focaliser à la fois en émission et en réception.

\subsection{Filtre adapté en réception.}

Lorsque les procédures classiques de focalisation en réception réalignent les signaux reçus en se référant à la loi de retards d'émission, elles réalisent en fait une convolution de l'écho reçu par cette loi. Nous proposons de convoluer l'écho du réflecteur (reçu après focalisation en émission) par un ensemble de signaux adaptés, soit la banque de données acquise dans la procédure d'émission (voir Figure 4). Considérant que le réflecteur idéal renvoie aux transducteurs un signal $r_{i}{ }^{\prime}\left(r_{0}, t\right)$ équivalent à $r_{i}\left(r_{0}, t\right)$, la convolution de cet écho par la banque de données donne sur chaque voie du réseau le signal

$$
\begin{aligned}
& r_{i}{ }^{\prime}\left(r_{0}, t\right) \otimes t_{i}\left(r_{0}, t\right) \\
= & r_{i}\left(r_{0}, t\right) \otimes t_{i}\left(r_{0}, t\right)
\end{aligned}
$$




$$
=r_{i}\left(r_{0}, t\right) \otimes r_{i}\left(r_{0}, T-t\right) .
$$

Ce signal est symétrique avec un maximum au temps $T$ quel que soit le transducteur considéré. La somme

$$
S\left(r_{0}, t\right)=\sum_{i} r_{i}\left(r_{0}, t\right) \otimes r_{i}\left(r_{0}, T-t\right)
$$

crée une interférence constructive optimale au temps $T$ pour un écho provenant de $r_{0}$. Cette focalisation est parfaitement adaptée au réflecteur et tient compte de tous les effets de propagation du milieu inhomogène. Ceci est confirmé par quelques expériences de focalisation en émission-réception.

\subsection{Résultats expérimentaux.}

Les mesures de diagrammes de directivité en émissionréception nécessitent de recueillir les échos d'un réflecteur scannant le plan focal. Toutefois, le réseau de transducteurs reste focalisé en émission sur la position $r_{0}$. Cette procédure est illustrée Figure 5 .

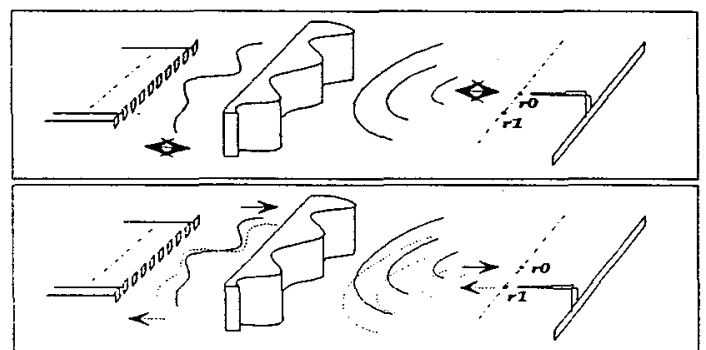

Fig. 5. Procédé expérimental d'acquisition de diagramme de directivité en émission-réception. Le réseau de transducteurs enregistre chaque écho provenant de chaque position du réflecteur.

Comme prévu, l'amplitude des signaux $S\left(r_{1}, t\right)$ chute fortement quand $\overline{\Gamma_{0} \Gamma_{1}}$ augmente. L'emploi d'un filtre adapté est pleinement justifié en permettant dorénavant d'atteindre des niveaux de lobes très satisfaisants. La Figure 6 présente la comparaison des diagrammes de directivité obtenus en émission-réception par retards adaptatifs et par retoumement temporel. $\mathrm{Si}$, pour le cas d'un aberrateur collé au réseau, la focalisation est équivalente, elle s'améliore nettement pour un aberrateur éloigné du réseau. La cause en est bien évidemment l'utilisation de la banque de données adaptée à la source échogène.

\section{Conclusion.}

D'un point de vue théorique, le filtre adapté optimise la focalisation en émission et en réception en fournissant la meilleure entrée possible au système représentatif du milieu inhomogène. Il permet une meilleure représentation du point réflecteur que les techniques de retards, où que soit situé l'aberrateur. L'interférence constructive obtenue par le principe du filtre adapté procure un niveau de lobes secondaires suffisamment bas pour considérer des applications d'imagerie médicale. Toutefois, elle nécessite dans les tissus la présence d'un réflecteur qui n'est pas toujours disponible. L'extension de ce concept à des milieux diffuseurs en lieu et place d'un réflecteur est actuellement à l'étude dans notre laboratoire.
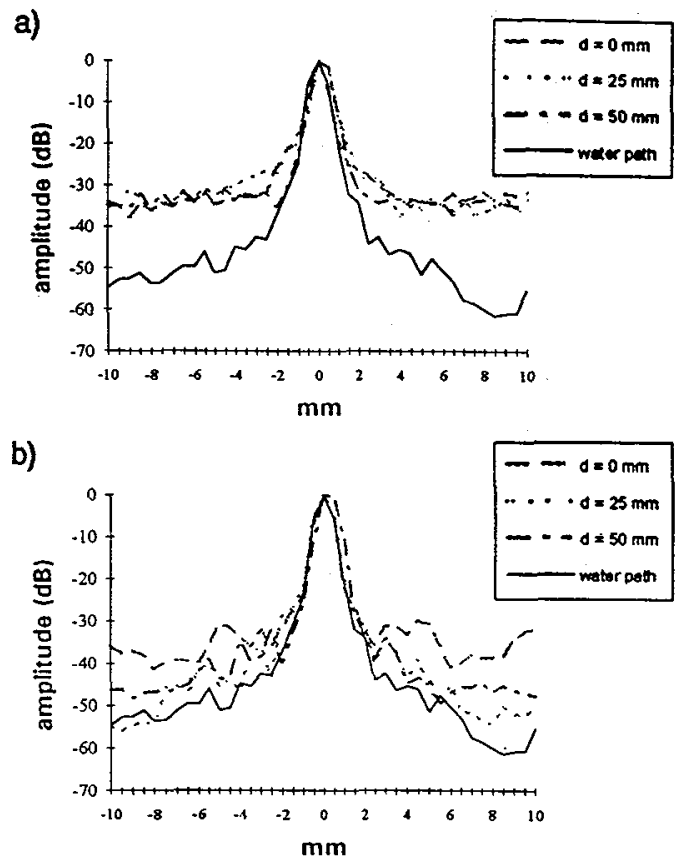

Fig. 6. Diagrammes de directivité en émission-réception par retards adaptatifs (a). Diagrammes de directivité en émissionréception par retoumement temporel (b).

\section{Référencess.}

[1] M. Fink, C. Prada, F. Wu, D. Cassereau. "Self focusing in inhomogeneous media with time reversal acoustic mirrors". in Proc. IEEE Ultras. Symposium (Montreal 1989) vol.2, pp. 681-686.

[2] L.Nock, G.E. Trahey, S.W. Smith. "Phase aberration correction in medical ultrasound using speckle brightness as a quality factor" J. Acoust. Soc. Am. vol.85, pp. 18191833 (1989).

[3] S.W. Flax, M. ODonnell. "Phase aberration correction using signals from point reflectors and diffuse scatterers: Basic principles". IEEE trans. UItras. Ferroelec. Freq. Con. vol 35,pp 758-767 (1988).

[4] F. Wu, M. Fink, R. Mallart, J.L. Thomas, N.Chakroun, D. Cassereau and C. Prada. "Optimal focusing through aberrating media: a comparison between time reversal mirror and time delay correction techniques", in Proc. IEEE Ultrason. Symp. 1991, pp. 1195-1199.

[5] M. Fink. "Time reversal of ultrasonic fields: Basic pinciples". IEEE trans. Ultras. Ferroelec. Freq. Con. vol 39, pp.555-567 (1992). 